12th World Computer Congress IFIP CONGRESS 92

7.-11. September 1992 - Madrid, Spain

Auskunft: Prof. Dr. Wilfried Brauer

TU München

Institut für Informatik

Postfach 202420

W-8000 München 2

\section{DGOR/ÖGOR Jahrestagung 92}

9.-11. September 1992 - Aachen

Auskunft: Prof. Dr. Karl-Werner Hansmann

Universität der Bundeswehr Hamburg

Institut für Industrielles Management

Holstenhofweg 85

W-2000 Hamburg 70

Tel.: 040/6541-2772

Kombinatorik

1.-7. November 1992 - Oberwolfach

Auskunft: Mathematisches Forschungsinstitut Oberwolfach

Prof. Dr. Martin Barner

Albertstr. 24

W-7800 Freiburg

Komplexitätstheorie

15.-21. November 1992 - Oberwolfach

Auskunft: Mathematisches Forschungsinstitut Oberwolfach

Prof. Dr. Martin Barner

Albertstr. 24

W-7800 Freiburg

Combinatorial Optimization

17.-23. Januar 1993 - Oberwolfach

Auskunft: Mathematisches Forschungsinstitut Oberwolfach

Prof. Dr. Martin Barner

Albertstr. 24

W-7800 Freiburg
Mathematische Stochastik

7.-13. März 1993 - Oberwolfach

Auskunft: Mathematisches Forschungsinstitut Oberwolfach Prof. Dr. Martin Barner

Albertstr. 24

W-7800 Freiburg

Combinatorial Convexity and Algebraic Geometry

28. März - 3. April 1993 - Oberwolfach

Auskunft: Mathematisches Forschungsinstitut Oberwolfach Prof. Dr. Martin Barner

Albertstr. 24

W-7800 Freiburg

IFORS - OR: Expanding Horizons

12.-16. Juli 1993 - Lissabon, Portugal

Auskunft: Prof. Dr. Wolfgang Bühler

Universität Mannheim

Lehrstuhl füt Finanzierung

Postfach

W-6800 Mannheim

Tel; (0621) 292-5446

Dynamische Systeme

18.-24. Juli 1993 - Oberwolfach

Auskunft: Mathematisches Forschungsinstitut Oberwolfach Prof. Dr. Martin Barner

Albertstr. 24

W-7800 Freiburg

22nd Conference on Statistics Processes and their Applications

21.-25. Juli 1993 - Amsterdam, The Netherlands

Auskunft: SPA'93, c/o CWI

P.O. Box 4079

1009 AB Amsterdam, The Netherlands

\title{
Liste der zur Besprechung eingegangenen Bücher
}

Die aufgelisteten Bücher sind zur Besprechung im OPERATIONS RESEARCH SPEKTRUM eingegangen. Die mit einem Stern versehenen Bücher können zur Besprechung bei Prof. Dr. W. Junginger, Universität der Bundeswehr Hamburg, Postfach 700822, 2000 Hamburg 70 , angefordert werden:

* Cambini, A., Castagnoli, E., Martein, L., Mazzoleni, P., Schaible, S.: Generalized Convexity and Fractional Programming with Economic Applications. Berlin, Heidelberg, New York: Springer 1990

* El-Hodiri, M. A.: Extrema of Smooth Functions. Berlin, Heidelberg, New York: Springer 1991. 197 S., DM 80,-

* Francis, McGinnis, White: Facility Layout and Location: An Analytical Approach. Englewood Cliffs, NJ: Prentice Hall 1992

Hildenbrandt, $W$., Sonnenschein, H.: Handbook of Mathematical Economics - Vol. 4. Amsterdam: Elsevier Science 1991. 723 pp., $\$ 82,50 /$ Dfl. 250 ,-
* Kosmol, Peter: Optimierung und Approximation. Berlin, New York: de Gruyter 1991. 394 S., DM 58,-

Trost, $R$.: Entscheidungen unter Risiko: Bernoulliprinzip und duale Theoric. Frankfurt/M., Bern, New York, Paris: Peter Lang 1991. 198 pp., DM 60,-

* Schneewei $\beta$, C.: Planung 1 -Systemanalytische und entscheidungstheoretische Grundlagen. Berlin, Heidelberg, New York: Springer 1991. 389 S., DM 32,80

* Flippo, O.E.: Stability, duality and decomposition in general mathematical programming. Amsterdam: CWI 1991. 361 pp., DM 69,- 